\title{
LOCALIZAÇÃO DE FALTAS EM LINHAS DE TRANSMISSÃO EM CORRENTE CONTÍNUA SEGMENTADAS
}

\author{
Eduardo Gonzaga da Silveira ${ }^{1}$ \\ Frederico José de Oliveira ${ }^{2}$
}

\section{RESUMO}

Este trabalho apresenta uma técnica para a localização de faltas em sistemas de transmissão High Voltage Direct Current (HVDC) constituídos por linhas aéreas e cabos isolados. O método foi baseado na coleta de dados de dois terminais nas extremidades do elo de corrente contínua e pela detecção de faltas através de coeficientes da Transformada Wavelet Discreta tipo Haar. A partir do problema proposto, foi simulado com o auxílio do Alternative Transient Program (ATP), um sistema de transmissão em corrente contínua monopolar com conversores a tiristor Current Source Converter (CSC), constituído de duas linhas aéreas de $150 \mathrm{~km}$ e $80 \mathrm{~km}$, e um segmento intermediário de cabo subterrâneo com $80 \mathrm{~km}$. Os dados das respostas transitórias de curtoscircuitos em diferentes segmentos do elo foram carregados em uma interface gráfica criada no Matrix Laboratory (MATLAB), para realizar as rotinas de pré-processamento, além de detectar e localizar a falta. O método foi testado para diferentes taxas de amostragem e impedâncias de falta, ele apresentou resultados satisfatórios na localização de faltas em todos os segmentos da linha, com erros em torno de $0,5 \%$ do comprimento total do elo, além do baixo custo computacional, o que mostra a viabilidade de sua implementação prática.

Palavras-chave: HVDC. Localização de faltas. ATP. Transformada Wavelet.

\section{LOCATION OF FAULTS IN TRANSMISSION LINES IN CONTINUOUS SEGMENTED STREAM}

\begin{abstract}
This paper presents a technique for location of faults in High Voltage Direct Current (HVDC) transmission systems constituted of overhead lines and cables. The method was based on data collection of two terminals on the extremity of the continuous stream link, and through the fault detection using the Discrete Wavelet Transform coefficients of Haar type. From the proposed problem, it was simulated, with the support of the Alternative Transient Program (ATP), a transmission system in monopolar continuous stream with thyristor converters Current Source Converter (CSC), constituted of two overhead lines of $150 \mathrm{~km}$ and $80 \mathrm{~km}$, and an intermediate

\footnotetext{
${ }^{1}$ Doutorado em Engenharia Elétrica pela Universidade Federal de Minas Gerais (2007), professor de Ensino Básico

${ }^{2}$ Graduando em Engenharia Elétrica pelo CEFET-MG. E-mail: frederico511@ hotmail.com
} 
segment of underground cable with $80 \mathrm{~km}$. The data of the transient responses of short circuits, in different segments of the link, were loaded in a graphical interface (GUI) created in Matrix Laboratory (MATLAB) to perform the routines of the pre-processing, besides detecting and localizing the fault. The method was tested for different sampling rates and fault impedances. It showed satisfactory results on the location of the faults in all segments of the line, with errors around $0.5 \%$ of the total length of the link, beyond the low computational cost, which shows the viability of its practical implementation.

Keywords: HVDC. Fault location. ATP. Wavelet Transform.

\section{INTRODUÇÃO}

Os sistemas elétricos de energia têm atualmente suas bases na corrente alternada. Entretanto, na atual fase de desenvolvimento, existem grandes centros de cargas distantes dos locais de geração de energia ou que precisam ser conectados a sistemas elétricos com frequências diferentes. Há casos também de cargas ou fontes que estão no meio do oceano e distantes da costa. Frente a esses desafios, os sistemas High Voltage Direct Current (HVDC) se tornaram uma solução flexível e confiável na transmissão de grandes blocos de energia elétrica, em função do desenvolvimento dos semicondutores de potência a partir da década de 60 , principalmente com o advento dos tiristores (ARRUDA, 2012).

Os elos de corrente contínua caracterizam-se pela interligação de dois sistemas de corrente alternada por meio de duas estações conversoras, sendo um terminal retificador e um inversor. A conexão entre estas estações é feita por uma ou mais linhas aéreas de transmissão e/ou cabos isolados, ou até mesmo, uma transmissão mista utilizando linhas e cabos, todos possuindo uma ou duas polaridades, ou seja, são construídos com retorno pela terra ou com a utilização de dois condutores (BORRÉ, 2006). Essa interligação constitui uma parte sensível do sistema, principalmente quando se trata de uma linha aérea, pois está sujeita a intempéries da natureza e até mesmo a vandalismo, o que gera um maior número de faltas que podem ser temporárias ou permanentes (ARRUDA, 2012).

Deve-se ressaltar que devido às várias possibilidades de configuração da linha de transmissão que conecta os dois extremos do sistema, o processo de localização de faltas se torna complexo, principalmente quando os surtos ocorrem em segmentos de cabos isolados. Assim, o 
problema proposto é caracterizado pela busca de meios para se conseguir a localização de faltas em toda a extensão da linha que interliga as estações retificadora e inversora, sejam estas compostas apenas de linhas e/ou com sessões possuindo cabos subterrâneos ou submarinos, em um caso mais geral.

A detecção e localização de faltas, principalmente em cabos subterrâneos e submarinos, são de grande importância, levando em consideração que as ações tomadas pelo sistema de proteção devem ser distintas se comparadas com uma linha aérea. Além disso, em situações faltosas, a equipe de manutenção saberá como agir, para que a conexão seja restabelecida o mais rápido possível (OLIVEIRA, 2013).

\section{LOCALIZAÇÃO DE FALTAS EM SISTEMAS HVDC}

Este trabalho propõe um método de localização de faltas que se enquadra na utilização de transitórios de alta frequência, obtidos dos dois terminais de medição localizados nas extremidades do elo, com o auxílio da Transformada Wavelet Discreta. Para esta técnica, a estimação da distância em que ocorreu a falta é, geralmente, independente da impedância de surto e de mudanças nas configurações das fontes dos sistemas de potência. A localização da falta apenas poderá ser determinada com boa exatidão se as medições em ambas as estações forem sincronizadas (PASSOS, 2010).

\subsection{Localização de faltas por meio da teoria de ondas viajantes}

Um tipo de distúrbio em uma linha de transmissão como uma descarga atmosférica ou uma interrupção nas condições de regime permanente, gera a propagação de ondas viajantes, que trafegam até os extremos da linha para então serem refletidas e refratadas. Assim, a teoria das ondas viajantes permite que sejam definidos os coeficientes de reflexão e refração da onda em descontinuidades, a velocidade de propagação da onda e a impedância de surto na linha de transmissão (VALINS, 2005).

Para uma linha com apenas um segmento, a localização da falta é encontrada por meio da equação 1.

$$
\mathrm{x}_{\mathrm{F}}=\frac{\left[\mathrm{x}-\Delta \mathrm{t} \cdot \mathrm{v}_{\mathrm{p}}\right]}{2}
$$


onde: $\mathrm{x}$ é o comprimento total da linha; $\Delta$ t é a diferença dos tempos de chegada das ondas nos dois terminais de medição; e $\mathbf{v}_{\mathbf{p}}$ é a velocidade de propagação da onda na rede, obtida por meio da indutância (L ) e da capacitância (C) por unidade de comprimento da linha, como mostrado na equação 2.

$$
\mathbf{v}_{\mathbf{p}}=\frac{1}{\sqrt{\mathrm{LC}}}
$$

\subsection{Extensão para sistema de transmissão HVDC com três segmentos}

Para um caso geral em sistemas HVDC, com elo misto de cabos isolados e linhas aéreas, a localização das faltas pode ser formulada de maneira que são levados em consideração as diferentes velocidades de propagação e os diferentes comprimentos de cada segmento. Na figura 1, tem-se um exemplo dos diversos casos de faltas que podem ocorrer em uma conexão de duas linhas aéreas nas extremidades e um cabo isolado na parte central, a qual foi adotada para a simulação.
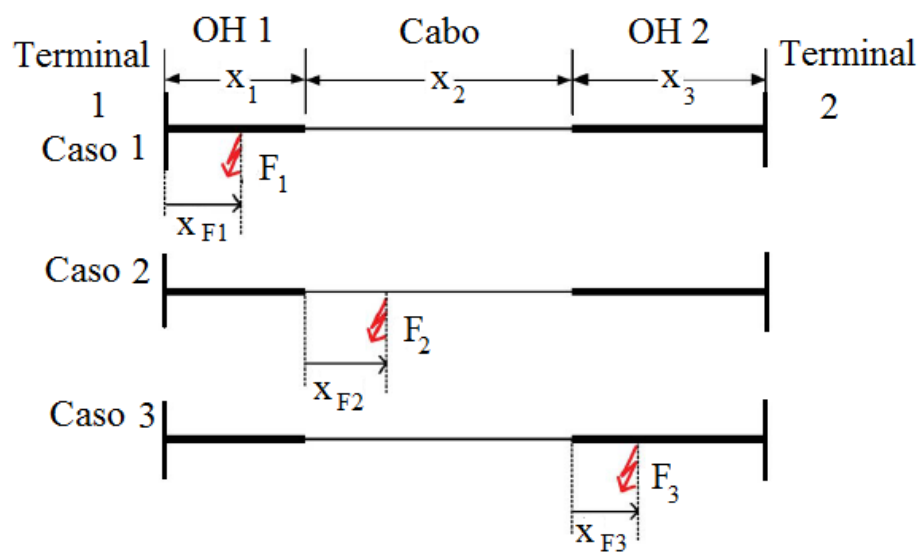

Figura 1 - Sistema HVDC com dois segmentos de linha aérea e um de cabo isolado Fonte: Nanayakkara, Rajapakse e Achal (2012).

Sejam $\mathbf{x}_{1}, \mathbf{x}_{2}$ e $\mathbf{x}_{3}$ os comprimentos dos três segmentos da linha na figura 1. Existem três casos: uma falta $\mathbf{F}_{1}$ localizada no segmento 1, a falta $\mathbf{F}_{2}$ ocorrendo na sessão 2 e $\mathbf{F}_{3}$ que pode ocorrer na terceira parte da linha como representado na figura 1. Pela análise do primeiro caso, define-se $\mathbf{x}_{\mathbf{F} 1}$ como a distância de ocorrência de $\mathbf{F}_{1}$ em relação ao terminal 1 (retificador). Para uma falta em $\mathrm{t}=0$, o tempo de chegada da onda viajante no terminal 1 pela equação 3 .

ForSci.: r. cient. IFMG campus Formiga, Formiga, v. 1, n. 1, p. 33-48, jul./dez. 2013. 


$$
t_{1-F 1}=\frac{x_{\mathrm{F} 1}}{v_{1}}(3)
$$

onde $\mathbf{v}_{1}$ é a velocidade de propagação da onda na sessão 1 . O tempo de chegada da onda no terminal 2 (inversor) é dado pela equação 4

$$
t_{2-F 1}=\left(\frac{x_{1}-x_{F 1}}{v_{1}}\right)+\frac{x_{2}}{v_{2}}+\frac{x_{3}}{v_{3}}(4)
$$

com $\mathbf{v}_{2}$ e $\mathbf{v}_{3}$ na equação 4 sendo as velocidades de propagação nos segmentos 2 e 3 respectivamente da figura 1 . A diferença entre os tempos de chegada aos terminais é dada pela equação 5.

$$
\Delta t_{12-F 1}=\frac{2 x_{F 1}}{v_{1}}-\frac{x_{1}}{v_{1}}-\frac{x_{2}}{v_{2}}-\frac{x_{3}}{v_{3}}
$$

A localização da falta começando a partir do início do segmento 1 é encontrada pela equação 6.

$$
\mathrm{x}_{\mathrm{F} 1}=\left(\Delta \mathrm{t}_{12-\mathrm{F} 1}+\frac{\mathrm{x}_{1}}{\mathrm{v}_{1}}+\frac{\mathrm{x}_{2}}{\mathrm{v}_{2}}+\frac{\mathrm{x}_{3}}{\mathrm{v}_{3}}\right) \frac{\mathrm{v}_{1}}{2}
$$

No segundo caso, tem-se a falta no segmento intermediário em $\mathrm{t}=0$ e $\mathbf{x}_{\mathrm{F} 2}$ representando a distância da falta medida a partir do início do segmento 2. Os tempos de chegada aos dois terminais são dados por pelas equações 7 e 8 respectivamente.

$$
\begin{gathered}
t_{1-F 2}=\frac{x_{F 2}}{v_{2}}+\frac{x_{1}}{v_{1}} \\
t_{2-F 2}=\left(\frac{x_{2}-x_{F 2}}{v_{2}}\right)+\frac{x_{3}}{v_{3}}
\end{gathered}
$$

Para o caso 2, a diferença entre os tempos de chegada é calculado por meio da equação 9.

$$
\Delta t_{12-F 2}=\frac{2 x_{F 2}}{v_{2}}+\frac{x_{1}}{v_{1}}-\frac{x_{2}}{v_{2}}-\frac{x_{3}}{v_{3}}
$$

ForSci.: r. cient. IFMG campus Formiga, Formiga, v. 1, n. 1, p. 33-48, jul./dez. 2013. 
A localização da falta é dada pela equação 10 .

$$
\mathrm{x}_{\mathrm{F} 2}=\left(\Delta \mathrm{t}_{12-\mathrm{F} 1}-\frac{\mathrm{x}_{1}}{\mathrm{v}_{1}}+\frac{\mathrm{x}_{2}}{\mathrm{v}_{2}}+\frac{\mathrm{x}_{3}}{\mathrm{v}_{3}}\right) \frac{\mathrm{v}_{2}}{2} \quad(10)
$$

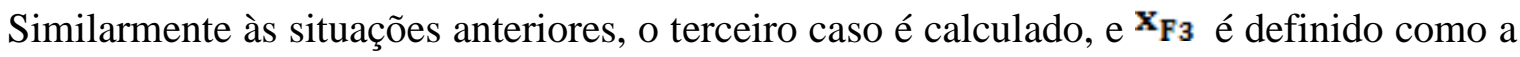
distância de localização da falta $\mathbf{F}_{3}$ medida a partir do início do segmento 3 , calculado pela equação 11:

$$
\mathrm{x}_{\mathrm{F} 3}=\left(\Delta \mathrm{t}_{12-\mathrm{F} 3}-\frac{\mathrm{x}_{1}}{\mathrm{v}_{1}}-\frac{\mathrm{x}_{2}}{\mathrm{v}_{2}}+\frac{\mathrm{x}_{3}}{\mathrm{v}_{3}}\right) \frac{\mathrm{v}_{3}}{2} \quad \text { (11) }
$$

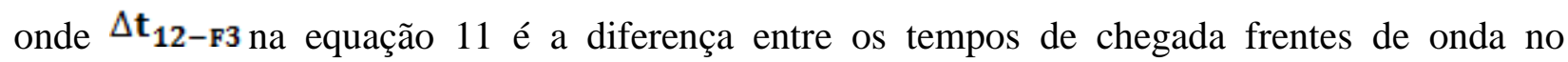
terminais de medição para o caso 3 .

Para estimar a localização da ocorrência da falta (NANAYAKKARA; RAJAPAKSE; ACHAL, 2012), é utilizado o algoritmo mostrado na figura 2. Primeiramente, a diferença entre os tempos de chegada das ondas é calculada e aplicam-se as equações (6), (10) e (11), obtendo-se três valores de distâncias. Estes valores passam por uma condição que define se eles correspondem ao tamanho do respectivo segmento em questão analisado. Em todos os casos, apenas uma comparação será verdadeira e o algoritmo utilizará esta distância para calcular a localização da falta, não promovendo ambiguidades nos resultados.

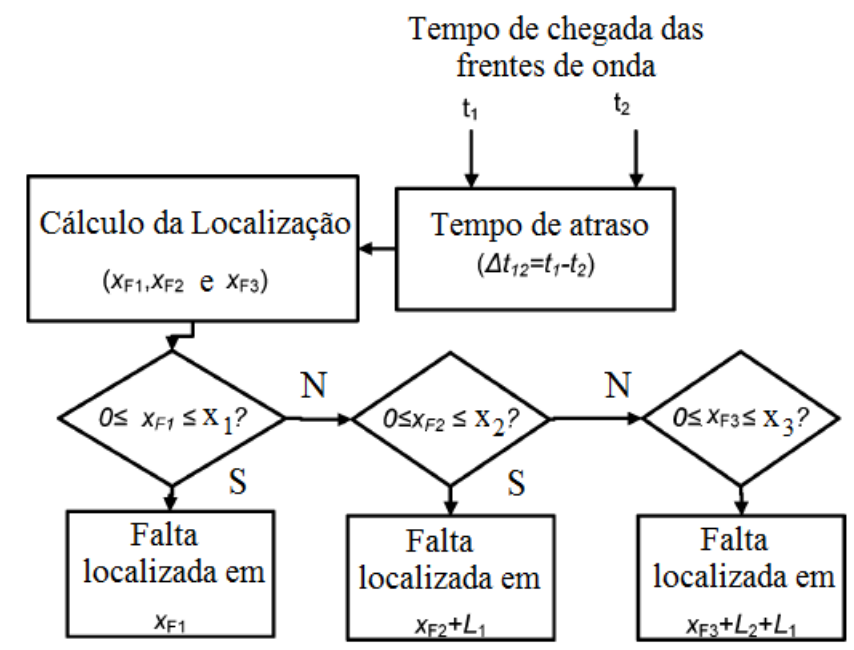

Figura 2 - Diagrama de blocos do algoritmo de localização de faltas para determinação do segmento faltoso Fonte: Autor 

segmentadas

Se for localizada uma falta no segmento 2, o tempo de atraso em relação a chegada das

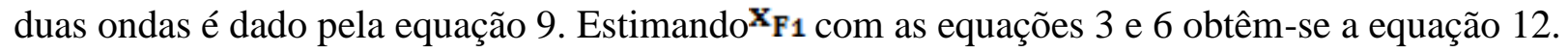

$$
\mathrm{x}_{\mathrm{F} 1}=\mathrm{x}_{1}+\mathrm{x}_{\mathrm{F} 2} \frac{\mathrm{v}_{1}}{\mathrm{v}_{2}}
$$

Pode-se dizer que a razão entre $\left(\mathbf{v}_{\mathbf{1}} / \mathbf{v}_{\mathbf{2}}\right)$ será sempre maior que zero. Logo, a falta não está na primeira sessão da linha, pois $\mathbf{x}_{\mathrm{F} 1}>\mathbf{x}_{1}$. Calculando por hipótese $\mathbf{x}_{\mathrm{F} 3}$ chaga-se na equação 13 .

$$
\mathrm{x}_{\mathrm{F} 3}=\left(\mathrm{x}_{\mathrm{F} 2}-\mathrm{x}_{2}\right) \frac{\mathrm{v}_{3}}{\mathrm{v}_{2}}
$$

Na equação 13, desde que $\left({ }^{\mathbf{v}_{3}} / \mathbf{v}_{2}\right.$ ) seja maior que zero e $\mathbf{x}_{\mathrm{F} 2}$ é menor que $\mathbf{x}_{\mathbf{2}}$, o valor de $\mathbf{x}_{\mathrm{F} 3}$ se torna negativo, o que impossibilita uma falta no terceiro segmento. Portanto, o método de detecção é uma ferramenta que não gera ambiguidades, a qual pode ser utilizada em um elo com $\sigma$ segmentos. Uma rede conceitual para o uso do algoritmo é mostrada na figura 3.

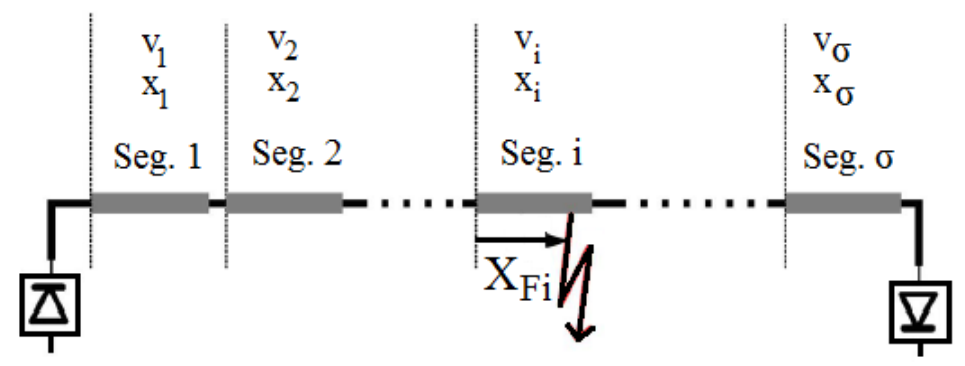

Figura 3 - Sistema HVDC genérico com rede de $\sigma$ segmentos Fonte: Nanayakkara, Rajapakse e Achal (2012).

A estimativa da localização da falta para uma linha com $\sigma$ segmentos é expressa pela equação 14 (NANAYAKKARA; RAJAPAKSE; ACHAL, 2012).

$$
\mathrm{x}_{\mathrm{Fi}}=\left(\Delta \mathrm{t}_{12-\mathrm{Fi}}-\frac{\mathrm{x}_{1}}{\mathrm{v}_{1}}-\frac{\mathrm{x}_{2}}{\mathrm{v}_{2}} \ldots+\frac{\mathrm{x}_{\mathrm{\sigma}-1}}{\mathrm{v}_{\mathrm{\sigma}-1}}+\frac{\mathrm{x}_{\sigma}}{\mathrm{v}_{\sigma}}\right)(14)
$$

ForSci.: r. cient. IFMG campus Formiga, Formiga, v. 1, n. 1, p. 33-48, jul./dez. 2013. 


\section{IDENTIFICAÇÃO DAS FRENTES DE ONDA}

\subsection{Transformada Wavelet}

Para detectar a ocorrência de faltas na linha de corrente contínua, é necessária uma ferramenta robusta o bastante para trabalhar com sinais de ampla banda de frequências, que são aperiódicos, os quais podem conter componentes senoidais e de impulso. Neste contexto, a Transformada Wavelet (TW) viabiliza tais requisições, com o emprego de funções que são locais em tempo e frequência (JENSE; COUR-HARBO, 2001).

\subsection{Transformada Wavelet Discreta}

A definição da TWD é dada pela equação 15

$$
\operatorname{TWD}(r, v)=\frac{1}{\sqrt{a_{0}^{r}}} \sum_{i} s(i) \Psi^{*}\left(\frac{v-i b_{0} a_{0}^{r}}{a_{0}^{r}}\right)(15)
$$

onde: $\Psi$ é a wavelet mãe e os parâmetros de escala e de translação "a" e "b" são funções de um

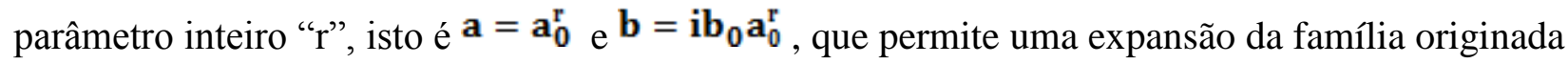
pela wavelet mãe, gerando as wavelets filhas. Na equação 15 , “v" é uma variável inteira que se refere a um número particular de amostras de um determinado sinal de entrada.

A saída da TWD pode ser representada em um espaço bidimensional de maneira semelhante à Transformada de Fourier Janelada Discreta, mas com divisões diferentes no tempo e frequência. A análise da TWD produz bandas de frequência de tempo retangulares, as quais são estreitas nas componentes mais oscilatórias e largas em componentes mais suaves. No entanto, as informações de alta frequência são as mais importantes para a detecção de faltas. Assim, um sinal pode ser dividido em duas partes, que são as aproximações, possuindo componentes de alta escala e baixa frequência do sinal, e os detalhes, os quais apresentam componentes de pequena escala e alta frequência.

Essa divisão é uma filtragem do sinal inicial s(t) mostrado na figura 4, utilizando um filtro passa-baixa e um passa-alta, os quais geram os vetores aproximação e detalhe com a metade do ForSci.: r. cient. IFMG campus Formiga, Formiga, v. 1, n. 1, p. 33-48, jul./dez. 2013. 
número de pontos do vetor inicial, com o auxílio do operador downsampling. Um exemplo de funcionamento (VALINS, 2005) da TWD em que foi considerada uma senoide com ruído de alta frequência como sinal de entrada é mostrado na figura 4.

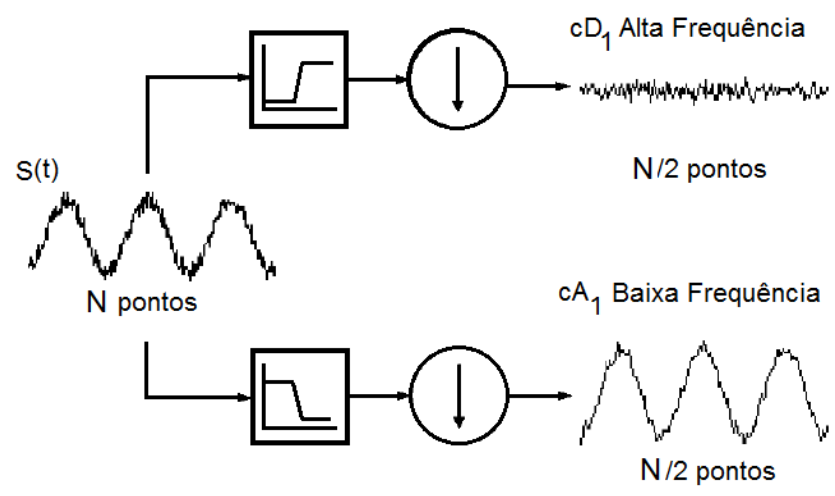

Figura 4 - Aplicação da TW em uma senoide acrescida de um ruído de alta frequência Fonte: Valins (2005).

\subsection{Wavelets de Haar}

A partir de uma pesquisa bibliográfica e testes realizados, foi constatado que a wavelet mãe mais adequada para a aplicação é a de Haar (OLIVEIRA, 2013). A detecção se dá por meio dos coeficientes de detalhe da TWD, os quais são analisados por uma função que estabelece um limiar de detecção. Caso seja processado um sinal de uma condição faltosa, o instante da falta é extraído pela janela de dados e enviado para o algoritmo de localização. É mostrado na figura 5 um exemplo da janela de processamento de um sinal. Na figura 6 é apresentado um resumo do algoritmo de detecção de faltas.

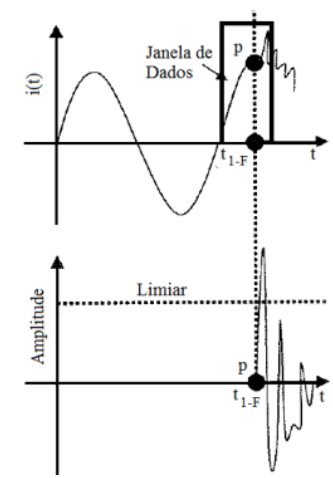

Figura 5 - Exemplo de janela de detecção do limiar Fonte: Autor

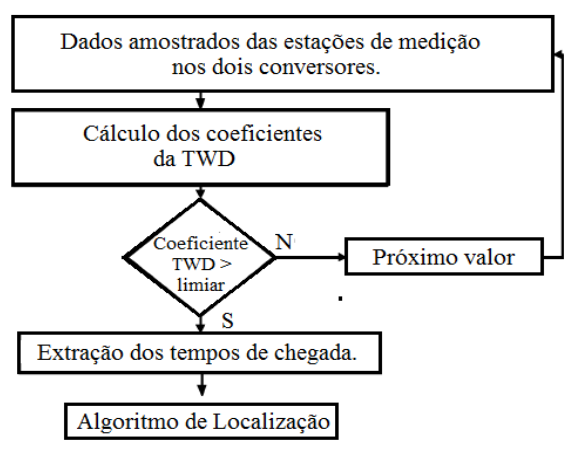

Figura 6 - Algoritmo de detecção Fonte: Autor

ForSci.: r. cient. IFMG campus Formiga, Formiga, v. 1, n. 1, p. 33-48, jul./dez. 2013. 


\section{DESCRIÇÃO DO SISTEMA SIMULADO E RESULTADOS OBTIDOS}

Por meio do Alternative Transient Program (ATP) foi construído um sistema HVDC Current Source Converter (CSC) na configuração monopolar com conversores de 12 pulsos, no qual foi modelada uma linha de transmissão com duas linhas aéreas nas extremidades, sendo a primeira com $150 \mathrm{~km}$, a segunda com $80 \mathrm{~km}$ e um cabo isolado na parte central com $80 \mathrm{~km}$ de extensão. A topologia monopolar se dá por um sistema de transmissão com apenas um condutor, sendo o retorno realizado pelo terra, com o aterramento da porção inferior das pontes retificadora e inversora. Uma visão geral do sistema é mostrada na figura 7 , onde são explicitadas as principais partes construtivas do sistema, como os transformadores, os terminais de inversão e de retificação, os filtros, os sistemas de medição de corrente e tensão na linha de transmissão.

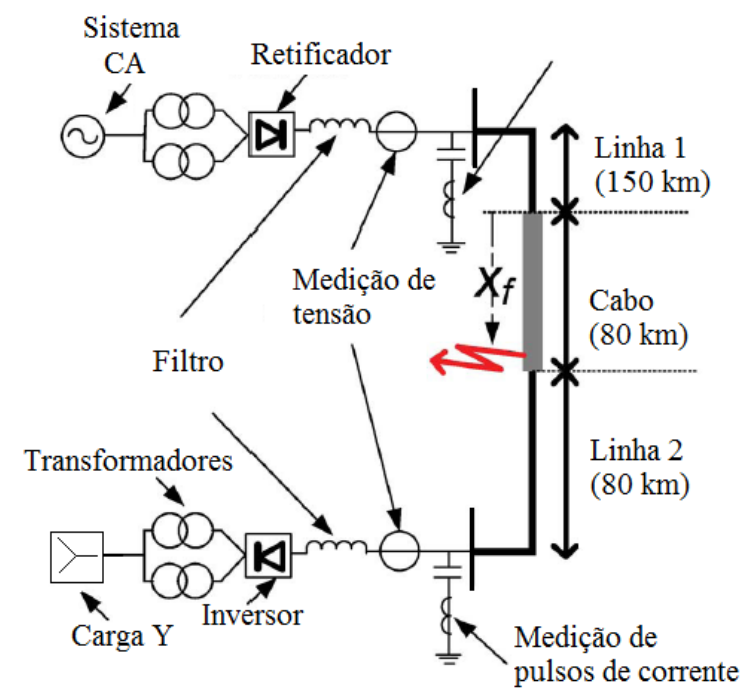

Figura 7 - Arquitetura geral do sistema HVDC construído Fonte: Nanayakkara, Rajapakse e Achal (2012).

\subsection{Parâmetros do sistema de transmissão}

Como mostrado na figura 7, o sistema de transmissão possui três segmentos. A tabela 1 exibe os parâmetros das linhas aéreas 1 e 2 simuladas no ATP, os quais foram obtidos por meio da rotina Line Constants no diretório LCC do ATP. Foi utilizado o modelo JMarti que possui seus parâmetros distribuídos e variáveis na frequência e mais precisos para linhas aéreas longas (OLIVEIRA, 2013). A resistividade do solo foi considerada de $1000 \Omega / \mathrm{m}$. 
SILVEIRA, E. G.; OLIVEIRA, F. J. Localização de faltas em linhas de transmissão em corrente contínua

Os parâmetros dos cabos de fase e guarda são mostrados na tabela 2 e a geometria da torre de transmissão utilizada para as duas linhas aéreas é exposta na figura 8.

Tabela 1 - Características e parâmetros das linhas 1 e 2

\begin{tabular}{ccc}
\hline Dado & Linha 1 & Linha 2 \\
\hline Número de fases & 1 & 1 \\
Cabo guarda & 1 & 1 \\
Tensão de operação & $500 \mathrm{kV}$ & $500 \mathrm{kV}$ \\
Modelo de simulação & JMarti & JMarti \\
Resistividade do solo & $1000 \Omega . \mathrm{m}$ & $1000 \Omega . \mathrm{m}$ \\
Frequência de cálculo & $0,05 \mathrm{~Hz}$ & $0,05 \mathrm{~Hz}$ \\
Comprimento & $150 \mathrm{~km}$ & $80 \mathrm{~km}$ \\
Resistência & $14,121 \Omega / \mathrm{km}$ & $27,937 \Omega / \mathrm{km}$ \\
Indutância & $1,6293 \mathrm{mH} / \mathrm{km}$ & $1,5897 \mathrm{mH} / \mathrm{km}$ \\
Capacitância & $8,5642 \mu \mathrm{F} / \mathrm{km}$ & $8,5642 \mu \mathrm{F} / \mathrm{km}$ \\
Velocidade da onda viajante & $2,6760 \times 10^{\mathbf{5}} \mathrm{km} / \mathrm{s}$ & $2,7091 \times 10^{5} \mathrm{~km} / \mathrm{s}$ \\
\hline
\end{tabular}

Fonte: Dados do autor

Tabela 2 - Dados dos condutores

\begin{tabular}{ccc}
\hline Dado & Fase & Guarda \\
\hline Rin $(\mathrm{m})$ & 0,5156 & 0 \\
Rout $(\mathrm{m})$ & 1,48 & 0,489 \\
Resis. $(\Omega / \mathrm{km})$ & 0,0611 & 1,490 \\
\hline
\end{tabular}

Fonte: Dados do autor

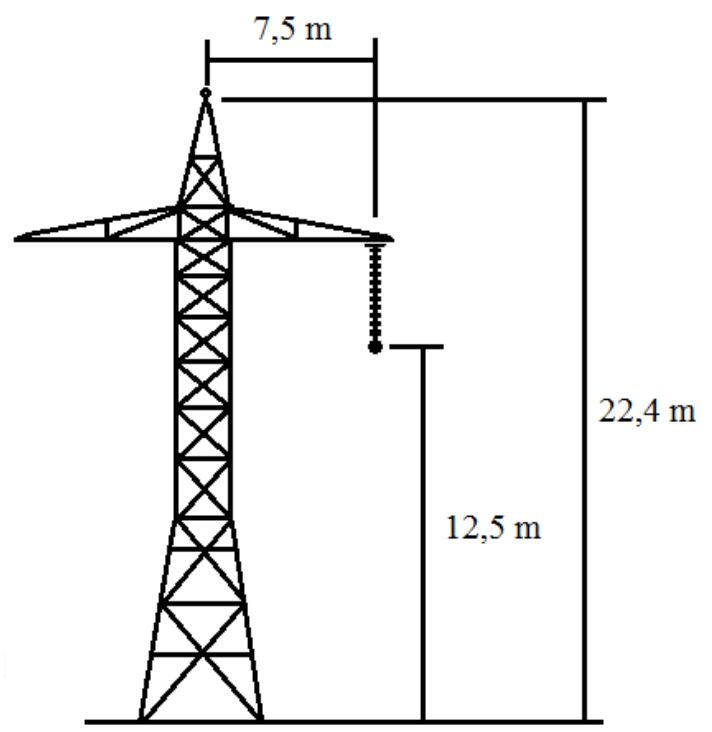

Figura 8 - Geometria da torre das linhas aéreas 1 e 2 Fonte: Autor

Na tabela 3, tem-se as características elétricas para a simulação do cabo subterrâneo. Foi utilizado o modelo Bergeron (OLIVEIRA, 2013) para cálculo dos parâmetros, o qual trabalha com parâmetros concentrados e variantes com a frequência.

ForSci.: r. cient. IFMG campus Formiga, Formiga, v. 1, n. 1, p. 33-48, jul./dez. 2013. 


\begin{tabular}{cc} 
Tabela 3 - Parâmetros do cabo isolado \\
\hline \multicolumn{2}{c}{ Características - Cabo Subterrâneo } \\
\hline Número de cabos & 1 \\
Tensão de operação & $500 \mathrm{~V}$ \\
Modelo de simulação & Bergeron \\
Resistividade do solo & $1000 \Omega . \mathrm{m}$ \\
Frequência de cálculo & $0,05 \mathrm{~Hz}$ \\
Comprimento & $80 \mathrm{~km}$ \\
Impedância & $4,8789 \mathrm{~m} \Omega / \mathrm{km}$ \\
Admitância & $204,96 \mathrm{~S} / \mathrm{km}$ \\
Impedância de surto & $39,124 \Omega$ \\
Velocidade da onda viajante & $1,8244 \times 10^{5} \mathrm{~km} / \mathrm{s}$ \\
\hline Fonte: Dados do autor
\end{tabular}

\subsection{Resultados das simulações}

Após a construção do sistema no ATP com a linha de transmissão mostrada na figura 7, foram simuladas faltas em todos os segmentos da rede e os dados dos dois terminais de medição foram exportados para uma interface gráfica criada no MATLAB, onde as funções de préprocessamento e algoritmo de detecção e localização foram implementadas. Tanto as impedâncias de falta como a taxa de amostragem das correntes foram variadas para analisar o comportamento do algoritmo de detecção e localização. O quadro 1 exibe os resultados obtidos nas simulações para cada impedância de falta e taxa de amostragem usada. Os campos que estão marcados com traço são aqueles que não obtiveram resultados satisfatórios. A equação 16 mostra como foi o procedimento para cálculo do erro, que é dado na ordem de quilômetro.

\section{$E(\mathbf{k m})=|\mathbf{k m}(\mathrm{TWD})-\mathbf{k m}(\mathbf{r e a l})| \quad(16)$}

Por meio da análise do quadro 1, observa-se que não houve significativo aumento dos erros quando a impedância foi maior, o que já era esperado por se tratar de um método de localização baseado em ondas viajantes. O melhor resultado obtido foi quando se utilizou uma impedância de falta nula e uma taxa de amostragem de $10 \mu$ s. Com um curto-circuito franco, as correntes de surto tiveram a amplitude do pico inicial aumentada, quando comparadas com as outras impedâncias simuladas, o que elevou o coeficiente da Transformada Wavelet e, portanto, o tempo de chegada da frente foi detectado com maior precisão.

ForSci.: r. cient. IFMG campus Formiga, Formiga, v. 1, n. 1, p. 33-48, jul./dez. 2013. 


\begin{tabular}{|c|c|c|c|c|c|c|c|c|c|}
\hline \multirow{2}{*}{$\begin{array}{c}\mathrm{km} \\
(\mathrm{real})\end{array}$} & \multicolumn{3}{|c|}{$1 \mu \mathrm{s}$} & \multicolumn{3}{c|}{$5 \mu \mathrm{s}$} & \multicolumn{3}{c|}{$10 \mu \mathrm{s}$} \\
\cline { 2 - 10 } & $\mathrm{Zf}=0 \Omega$ & $\mathrm{Zf}=10 \Omega$ & $\mathrm{Zf}=30 \Omega$ & $\mathrm{Zf}=0 \Omega$ & $\mathrm{Zf}=10 \Omega$ & $\mathrm{Zf}=30 \Omega$ & $\mathrm{Zf}=0 \Omega$ & $\mathrm{Zf}=10 \Omega$ & $\mathrm{Zf}=30 \Omega$ \\
\cline { 2 - 10 } & $\begin{array}{c}\text { Erro } \\
\mathrm{km})\end{array}$ & $\begin{array}{c}\text { Erro } \\
(\mathrm{km})\end{array}$ & $\begin{array}{c}\text { Erro } \\
(\mathrm{km})\end{array}$ & $\begin{array}{c}\text { Erro } \\
(\mathrm{km})\end{array}$ & $\begin{array}{c}\text { Erro } \\
(\mathrm{km})\end{array}$ & $\begin{array}{c}\text { Erro } \\
(\mathrm{km})\end{array}$ & $\begin{array}{c}\text { Erro } \\
(\mathrm{km})\end{array}$ & $\begin{array}{c}\text { Erro } \\
(\mathrm{km})\end{array}$ & $\begin{array}{c}\text { Erro } \\
(\mathrm{km})\end{array}$ \\
\hline 0 & - & - & - & 1,24 & - & - & 2,57 & - & - \\
\hline 30 & 0,92 & 0,92 & 0,99 & 0,66 & 0,66 & 0,66 & 0,66 & 0,66 & 0,66 \\
\hline 60 & 1,75 & 2,56 & 2,30 & 1,22 & 1,22 & 1,22 & 1,22 & 1,22 & 1,22 \\
\hline 90 & 2,58 & 2,58 & 3,39 & 1,78 & 1,88 & 3,12 & 0,89 & 0,89 & 0,89 \\
\hline 120 & 3,95 & 3,68 & 3,68 & 2,34 & 2,34 & 2,34 & 0,63 & 2,34 & 2,34 \\
\hline 150 & 3,98 & 2,18 & - & 2,90 & 2,90 & 4,25 & 2,90 & 2,90 & 2,90 \\
\hline 190 & 1,67 & 1,67 & 1,67 & 1,41 & 2,74 & 2,74 & 0,22 & 0,07 & 2,75 \\
\hline 230 & 3,06 & 3,06 & 0,11 & 2,53 & 2,53 & 0,64 & 0,64 & 0,64 & 0,64 \\
\hline 260 & 0,22 & 0,22 & 0,05 & 0,32 & 0,32 & 0,32 & 1,02 & 1,02 & 1,02 \\
\hline 290 & - & - & - & 0,88 & 0,88 & 0,88 & 0,46 & 0,46 & 2,21 \\
\hline 310 & - & - & - & - & - & - & - & - & - \\
\hline
\end{tabular}

Quadro 1 - Resultados para taxa de amostragem de 1 e $10 \mu \mathrm{s}$

Fontes: Dados do autor

Em relação ao tempo de amostragem, quando foi utilizada a taxa de $10 \mu \mathrm{s}$, foram obtidos maiores coeficientes da Transformada Wavelet. Como a transformada discreta analisa ponto a ponto do sinal de corrente, um maior intervalo de amostragem significa uma maior variação da amplitude da onda na situação de falta, portanto, o processamento de dados exibe coeficientes mais altos nestes casos, facilitando a localização.

$\mathrm{Na}$ maioria das vezes, o sistema de localização falhou na detecção das faltas nas subestações inversora e retificadora. Retornando à equação 6, nota-se que se a falta ocorrer no retificador, o tempo de chegada $\mathbf{t}_{1-F}$ será quase nulo, pois na simulação a falta é alocada sobre o sistema de medição que vem logo depois da subestação. O tempo de chegada aos terminais da subestação inversora será máximo e, portanto, o resultado da localização tenderá a ser negativo, o que é inválido em se tratando de comprimento.

ForSci.: r. cient. IFMG campus Formiga, Formiga, v. 1, n. 1, p. 33-48, jul./dez. 2013. 
A localização efetiva de faltas na subestação retificadora ocorreu quando se utilizaram taxas de amostragem de 5 e $10 \mu \mathrm{s}$, fazendo com que $\mathbf{t}_{1-\mathrm{F}}$ não ficasse tão próximo de zero. Não houve nenhum resultado válido para a localização de faltas na subestação inversora. Todos os valores encontrados resultaram acima do comprimento total da linha.

Os maiores erros encontrados são de faltas localizadas no cabo isolado e em suas proximidades. Pela análise da figura 9, percebe-se que a região de maior incerteza na localização das faltas encontra-se no segmento médio da linha. Este efeito pode ser explicado pela reflexão e transmissão das ondas viajantes perante descontinuidades, como é o caso da interface da linha aérea com o cabo isolado. Como as impedâncias características de ambos os segmentos são distintas, quando se considera uma falta em qualquer interface, uma pequena parcela do módulo da onda chega aos terminais de medição das subestações. Esta situação pode gerar ainda mais distorção e atenuação na onda e, consequentemente, maior erro na localização da falta.
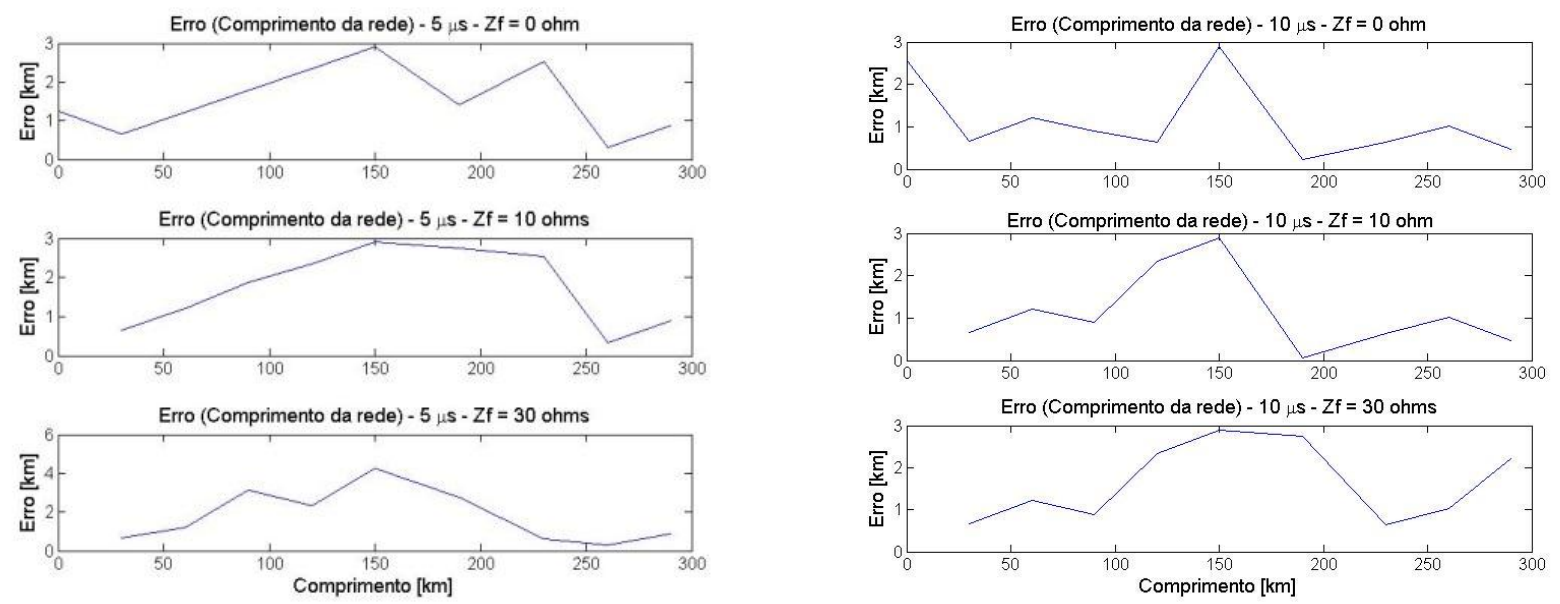

Figura 9 - Erro na localização das faltas em relação ao comprimento da linha Fonte: Dados do autor

\section{CONCLUSÕES}

O algoritmo proposto apresentou uma boa precisão na localização dos casos de falta testados, apresentado erros da ordem de 0,5\% do comprimento total da linha. Mesmo em situações que envolviam impedâncias de faltas mais elevadas, o algoritmo manteve uma precisão satisfatória. Para os casos testados, o algoritmo apresentou uma pequena influência na localização de faltas de acordo com o ponto de ocorrência da falta ao longo da linha de transmissão. Por meio 
dos resultados exibidos no quadro 1, nota-se que existe um ligeiro aumento do erro quando são simuladas faltas nas proximidades do cabo isolado, situação que mereceu uma análise mais profunda das características de reflexão e transmissão das ondas viajantes de corrente ao longo da linha.

Em relação às taxas de amostragem utilizadas, a taxa de $10 \mu \mathrm{s}$ obteve os melhores resultados. Essa característica se mostrou como um diferencial desta abordagem em relação aos algoritmos que utilizam dados de dois terminais da linha de transmissão na estimação da posição da falta. Em geral, estes apresentam uma grande influência da taxa de amostragem em seus resultados, apresentando maiores imprecisões quando se utilizam intervalos maiores de amostragem. Neste caso, a diminuição da frequência amostral aumentou os coeficientes da Transformada Wavelet, o que facilitou a localização dos pulsos de corrente em condições faltosas. Esse fato é de grande importância no caso da utilização do algoritmo de localização em conjunto com sistemas de proteção. A utilização de taxas de amostragem menores representa a utilização de processadores mais simples, o que resulta em um de processamento de dados em tempo relativamente menor.

Em aspectos gerais, com relação às sub-rotinas implementadas no algoritmo de localização, uma boa precisão e aplicabilidade do método utilizado foi alcançada quando se avalia os resultados das simulações. Um aspecto importante da abordagem utilizada é o fato de possuir um equacionamento simples, não necessitando de técnicas iterativas ou de recursos de hardware onerosos na obtenção dos resultados. Isso fica claro quando se avalia os cálculos envolvidos no processo de localização, sendo necessário para isso, apenas a manipulação algébrica de matrizes e vetores, através da Transformada Wavelet Discreta de Haar.

O método proposto, embora tenha sido testado em um sistema de transmissão em corrente contínua, pode ser utilizado em sistemas que operam em corrente alternada, pois os dados de entrada não dependem diretamente da frequência de operação da linha, apenas das características e parâmetros elétricos dos segmentos da rede em questão. Como a maioria dos algoritmos que utilizam dados de dois terminais necessita de fasores e/ou do ângulo de incidência da falta, isso constitui mais uma vantagem da abordagem realizada. 


\section{REFERÊNCIAS}

ARRUDA, F. M. O. Localização de faltas em sistemas de transmissão de faltas em sistemas de transmissão em corrente contínua (HVDC) utilizando Transformada Wavelet. 2012. 129f. Dissertação (Mestrado em Engenharia Elétrica) Centro Federal de Educação tecnológica de Minas Gerais. Belo Horizonte; CEFET MG, 2012.

BORRÉ, A. C. Bidirecionamento de Potência no TAP HVDC. 2006. 101f. Dissertação (Mestrado em Engenharia Elétrica), Universidade Federal do Rio de Janeiro (UFRJ). Rio de Janeiro: UFRJ, 2006.

JENSE, A.; COUR-HARBO, A. Ripples in mathematics: the Wavelet transform. New York: Springer, 2001.

NANAYAKKARA, O. M. K. K.; RAJAPAKSE, A. D.; ACHAL, R. Location of DC line faults in conventional HVDC systems with segments of cables and overhead lines using terminal Measurements. IEEE Transactions on Power Delivery, v. 27, n. 1, p. 279-288, jan. 2012.

OLIVEIRA, F. J. Localização de faltas em links de corrente contínua com cabos e linhas aéreas para sistemas HVDC. 2013. 153 f. Relatório (Iniciação Científica PIBIC/FAPEMIG), Centro Federal de Educação Tecnológica de Minas Gerais (CEFET MG). Belo Horizonte: CEFET MG, 2013.

OLIVEIRA, H. M. Análise de sinais para engenheiros: uma abordagem via Wavelets. São Paulo: Manole, 2003.

PASSOS, F. O. P. Solução para localização de faltas em linhas de transmissão não transpostas baseada em sincrofasores e algoritmo genético. 2010. 105f. Dissertação (Mestrado em Engenharia Elétrica) Universidade Federal de Itajubá (Unifei). Itajubá: Unifei, 2010.

VALINS, T. F. Relé digital de distância baseado na teoria de ondas viajantes e Transformada Wavelet. 2005. 143f. Dissertação (Mestrado em Engenharia Elétrica), Universidade Federal de São Carlos (UFSCAR). São Carlos: UFSCAR, 2005.

Recebido em: 15/09/2013

Aprovado em: 20/10//2013 